are not given in the review, but the "silly assertion" is, perhaps, a case of misunderstanding. Philosophers have debated whether the notion of 'an achieved infinity,' is a meaningful concept since Aristotle questioned the notion in his Phyics; see Sorabji ${ }^{2}$ for a discussion and a history of the debate up to the Middle Ages. The debate is still current; Popper and Wittgenstein ${ }^{3}$ had a sharp exchange on the question in 1946.

As one will see in the context of the book, we meant by an 'achieved infinity' a spacetime singularity. The Penrose diagram, or more generally the c-boundary construction, allows us to define a spacetime singularity in a mathematically precise way ${ }^{4}$ by attaching a boundary to spacetime. The c-boundary construction is very elegant and natural for globally hyperbolic spacetimes, the only class of spacetimes we considered. In the cboundary construction, a regular spacetime point is identified with the past light cone $I^{-}(\gamma)$ of a future-directed time-like curve $\gamma$ that terminates in that point, and the future c-boundary points are identified with the past light cones $I^{-}(\gamma)$ of future-directed time-like curves that have no future endpoints in the spacetime. (Past c-boundary points are defined analogously using future light cones.) If a time-like curve defining the c-boundary point is incomplete (that is, of finite length) the point is said" to be a 'singular c-boundary point'. These future singular c-boundary points are the future singularities.

It is generally accepted among relativists that if in fact the Universe is closed and terminates in a final singularity in finite proper time, then the Universe will actually reach this singularity. Strictly speaking, this is not true, since the c-boundary points are not in the spacetime, but there seems to be a general consensus to stretch the meaning of the word 'reach' in this case. Thus the final singularity really exists if the model of gravitational collapse is accurate. From the point of view of the c-boundary construction, the ontological status of the regular spacetime points and the future c-boundary points is the same: they are both the past light cones of timelike curves. Thus if one set of points really exist, then so does the other, and we agree that spacetime really exists. Furthermore, if the initial c-boundary of the Universe is an initial singularity, we can receive information from this singularity just as we can receive information from any other regular spacetime point in our past light cone. Thus it seems reasonable to say it really exists, if such is the initial c-boundary.

The singular c-boundary points are reasonably thought of as infinite because they are (in general) points at which physical quantities are actually infinite. Thus spacetime singularities - if they are indeed on the c-boundary of the actual Universe - are an achieved infinity and are

SCIENTIFCCORRESPONDENCE

precisely defined by the Penrose construction. I would have thought that this assertion is the overwhelming consensus of the relativity community.

Frank J. Tipler Departments of Mathematics and Physics, Tulane University, New Orleans,

Louisiana 70118, USA

1. Press, W.H. Nature 320, 315-316 (1986)

2. Sorabji, R. Time, Creation, and the ContinuumCh.14 (Duckworth, London 1983)

. Popper, K. Unended Quest: An Intellectual Autobiography, 15-16;123 (Fontana, London, 1976).

. Hawking, S.W. \& Ellis, G.F.R. The Large Scale Structure of Space-Time (Cambridge University Press, 1973).

5. Penrose, R. in Theoretical Principles in Astrophysics and Relativity (eds Lebovitz, N.R., Reid, W.H. \& Vandervoort, P.O.) 217-243 (University of Chicago Press, 1978).

\section{Estimating guild sizes}

SIR-John Lawton' has drawn attention in News and Views to the important simulation model of Shorrocks and Rosewell ${ }^{2}$ which assumes that aggregation of individual Drosophila species is reflected through the parameter $k$ of the negative binomial distribution. The model is based on successive sums of such distributions for two species. The resulting summed distribution is assumed to be negative binomial also, with parameter $k_{s}$, equal to the sum $k_{1}+k_{2}$ of those of the component species.

Shorrocks and Rosewell correctly note that this is precisely true only if $\mu_{1} k_{2}=$ $\mu_{2} k_{1}$, but claim that with equal mean values of 10 or more and values of $k<2$, it is a close approximation. This claim is technically wrong. Assuming the summed distribution is approximately negative binomial, and that the component species have equal mean values, the method of moments estimator of $k_{s}$ may easily be found to be

$$
\hat{k}_{s}=\frac{4 k_{1} k_{2}}{k_{1}+k_{2}}
$$

The approximation does not depend upon the mean value, and the mean value does not need to be 10 or more.

More importantly, however, unless $k_{1}+$ $k_{2}$ is close to $\hat{k}_{\text {s }}$ the approximation will be poor. To see the scale of error involved take two examples: first, $k_{1}=7 / 4, k_{2}=1 / 4$ gives a claimed $k_{s}$ of 2 compared with estimated $\hat{k}_{s}$ of $7 / 8$; second, $k_{1}=2, k_{2}=1 / 2$ gives a claimed $k_{\mathrm{s}}$ of $5 / 2$ compared with estimated $\hat{k}_{s}$ of $8 / 5$. Both examples show the approximation is not at all close. The effect that this and the assumption of equal mean values will have on the model requires thorough investigation, although it appears the correction would generate larger estimates of guild size than those estimated by Shorrocks and Rosewell which, as the authors themselves noted, were not quite as large as values from their field studies.

\section{Rothamsted Experimental Station}

Joe Perry

\section{Harpenden, Hertfordshire AL5 2JQ, UK}

1. Lawton, J. Nature 323, 398 (1986).

2. Shorrocks, B. \& Rosewell, J. J. anim. Ecol. 55, 527 (1986)

\section{Aluminium leaching from} cooking utensils

SIR-Aluminium is known to have neurotoxic effects ${ }^{1-3}$. With the discovery that abnormally high levels of aluminium are present in senile plaques in Alzhimer's dementia $^{3-5}$, the cumulative effects of aluminium poisoning and the question of how this metal enters the body become problems that need immediate attention. Recently, Coriat and Gillard have drawn attention to the high aluminium content of tea leaves and their infusions; aluminium compounds can also be found in water and can be released from utensils during cooking ${ }^{3-7}$. We have found that the leaching of aluminium from utensils is dramatically enhanced in the presence of trace quantities of fluoride ion.

In an experiment conducted to estimate the rate of leaching, we have found that the presence of only 1 p.p.m. of fluoride (the permitted level of fluoridation ${ }^{8}$ ) in water adjusted with citric acid or sodium bicarbonate to $p \mathrm{H} \sim 3$ (a $p \mathrm{H}$ often realized in cooking conditions) and boiled in an aluminium vessel, liberates nearly 200 p.p.m. of aluminium in $10 \mathrm{~min}$, compared with less than 0.2 p.p.m. in the absence of fluoride. Prolonged boiling produces a concentration of $\sim 600$ p.p.m., which is reached more quickly the larger the surface-to-volume ratio of the water. The rate of dissolution is $p \mathrm{H}$-dependent with a minimum at neutral $p \mathrm{H}$. Crushed tomatoes $(50 \mathrm{~g}$ in $250 \mathrm{ml})$ cooked in the same vessel with 1 p.p.m. of fluoride produced a concentration of $\sim 150$ p.p.m. of aluminium in 10 minutes. Water consumed in some localities contains 10 p.p.m. or more of fluoride. And cooking or prolonged storage in aluminium ware of foods that contain large amounts of fluoride ${ }^{9}(\sim$ 500 p.p.m. in tea, $100-700$ p.p.m. in fish) could easily release more than 100 p.p.m. of aluminium.

The normal resistance of aluminium to corrosion in mildly acidic or alkaline solutions depends on the formation of an inert oxide film. The corrosion in the presence of fluoride perhaps results from a permeability of the oxide film to fluoride ions, which disrupt the protective film.

K. TENNAKONE

S. WICKRAMANAYAKE

Institute of Fundamental Studies,

Hantana, Kandy, Sri Lanka and Department of Physics, University of Ruhuna, Matara, Sri Lanka

Wils. M.R. \& Savory, J. Lancet ii. 27 - 35 (1983)

. Brusewitz, S. Aluminium, University of Stockholm Institute of Physics Report 18 - 11 (1984)

3. Wurtman, R.J. Sci. Am. 252, 48 - 56 (1986)

. Duckett, S. \& Galle, P. C.r. hebd. Séanc. Acad. Sci. Paris 282, 393 - 395 (1976)

. Candy. J.M. et al. Lancet i, $354-357$ (1986)

6. Coriat, A.M. \& Gillard, R.D. Nature 321, $570(1986)$

7. Batchelor. B. et al. Envir. Sci. Technol. 20.891 - 894 (1986).

8. Pike, R.L. \& Brown, M.L. Nutrition: An Integrated Approach 3rd edn. 190 - 191 (Wiley, New York 1984)

9. Aswathnarayana, U. et al. Proc. Int. Symp. Geochem. Health (Royal Society, London, 1985 . 